\title{
Bone and cartilage in osteoarthritis: is what's best for one good or bad for the other?
}

\author{
Steven R Goldring and Mary B Goldring* \\ See related research by Bellido et al., http://arthritis-research.com/content/12/4/R152
}

\begin{abstract}
The interest in the relationship between articular cartilage and the structural and functional properties of peri-articular bone relates to the intimate contact that exists between these tissues in joints that are susceptible to the development of osteoarthritis (OA). The demonstration in several animal models that osteoporosis and decreased bone tissue modulus leads to an increased propensity for the development of post-traumatic OA is paradoxical in light of the extensive epidemiological literature indicating that individuals with high systemic bone mass, assessed by bone mineral density, are at increased risk for OA. These observations underscore the need for further studies to define the pathophysiological mechanisms involved in the interaction between subchondral bone and articular cartilage and for applying this information to the development of therapeutic interventions to improve the outcomes in patients with OA.
\end{abstract}

In a recently published manuscript, Bellido and coworkers have utilized an in vivo animal model to investigate the relationship between systemic osteoporosis and susceptibility to the development of post-traumatic osteoarthritis (OA) [1]. The interest in the relationship between articular cartilage and the structural and functional properties of peri-articular bone relates to the intimate contact that exists between these tissues in joints that are susceptible to the development of OA. Much of the interest and continuing controversy regarding the relationship between subchondral bone architecture and properties dates back to the original suggestion by Radin and Rose that alterations in the mechanical properties of subchondral bone could adversely affect the

*Correspondence: GoldringM@HSS.edu

Research Division, The Hospital for Special Surgery, Weill Cornell Medical College, New York, NY 10021, USA functional state of chondrocytes and the integrity of the overlying cartilage [2]. They hypothesized that the deterioration in articular cartilage associated with the osteoarthritic process was secondary to an initial increase in subchondral bone stiffness induced by repetitive mechanical loading.

Two general strategies have been used to define the properties of peri-articular bone in both human and animal models of OA $[3,4]$. The first strategy involves the use of nondestructive imaging modalities, including a variety of radiographic techniques, magnetic resonance imaging, radionuclide scanning and bone densitometry. A general conclusion from these studies is that the structural and organizational properties of the subchondral bone are dependent upon the stage of OA progression; that is, preclinical, early or late. Additionally, the alterations in the peri-articular bone are site specific, and it is essential to distinguish between the individual bone compartments of subchondral cortical and trabecular bone, as well as the zone of calcified cartilage.

In general, analyses using imaging techniques have confirmed that OA progression is associated with an increase in subchondral cortical bone volume and that these changes account for the pattern of subchondral sclerosis detected with standard radiographic techniques [5]. In contrast, analysis of the bone mineral density in human subjects with OA reveals a decrease in bone density related to thinning and loss of bone trabeculae [6]. These changes have been attributed to the effects of enhanced absorption of load-bearing stress by the thickened cortical bone plate, resulting in reduced load transfer to the underlying trabecular bone and the development of osteoporotic changes [7].

The second approach for analysis of peri-articular bone involves the analysis of subchondral bone specimens retrieved from human subjects or animal models at various stages of OA progression $[3,4]$. Particularly informative have been the studies by Day and colleagues [8], who utilized finite element models constructed from microCT scans of subchondral trabecular bone from the proximal tibiae of cadaver specimens of subjects with early OA. They observed that the volume fraction of 
trabecular bone was increased, but surprisingly found that the bone tissue modulus at sites corresponding to OA cartilage changes was reduced. They attributed the reduction in elastic modulus to a decrease in mineral density secondary to incomplete mineralization associated with an increased rate of bone remodeling.

The demonstration that OA, at least in the early stages, may be accompanied by a decrease in bone tissue modulus has direct relevance to the studies by Bellido and colleagues [1]. These authors employed a rabbit model in which they induced systemic osteoporosis with a protocol of ovariectomy and concomitant administration of glucocorticoids. Post-traumatic OA was induced using a surgical model, and a variety of investigative tools were employed to show that the induction of systemic and subchondral osteoporosis and increased bone remodeling resulted in enhanced cartilage damage compared with control animals. It is of interest that the studies by Day and colleagues also indicate that, although the bone volume is increased in specimens of subchondral bone from human subjects with early OA, there is a decrease in the elastic tissue modulus [8], similar to the effects of osteoporosis on bone mechanical properties.

The observation that osteoporotic bone changes may enhance rather than decrease the susceptibility to OA is paradoxical in light of the extensive literature in which the relationship between these two disorders has been investigated. In general, cross-sectional studies indicate that individuals with high systemic bone mass, assessed by bone mineral density, are at increased risk for the incidence of OA [4]. The apparent discrepancies in the data from these epidemiologic studies and from the studies of Bellido and colleagues [1] and Day and colleagues [8] highlight the complexity of the relationship that exists between subchondral bone and articular cartilage, which varies substantially depending on the stage of $\mathrm{OA}$ progression and the underlying factors involved in the pathogenesis of OA.

In the study by Bellido and colleagues, osteoporosis was induced using a combined protocol involving ovariectomy and glucocorticoids [1], both of which adversely affect bone remodeling. There are epidemiologic data in human subjects and data from animal models indicating that estrogen withdrawal is associated with an increased propensity for the development of OA, but whether these effects are mediated via direct action of estrogen on chondrocyte function or by effects on bone remodeling (or both) has not been established $[9,10]$. In addition, glucocorticoids also affect chondrocyte function, which could contribute to adverse effects on the state of the cartilage matrix [3]. Bellido and colleagues' study nevertheless provides additional insights into the complex relationship between peri-articular bone remodeling and the changes in articular cartilage during the evolution of $\mathrm{OA}$, and underscores the need for further work to define the optimal approaches for applying these insights into the development of therapeutic interventions that target the skeleton to improve the outcome in patients with OA.

\section{Abbreviations}

OA, osteoarthritis.

\section{Competing interests}

The authors declare that they have no competing interests.

\section{Published: 19 October 2010}

\section{References}

1. Bellido M, Lugo L, Roman-Blas JA, Castaneda S, Caeiro JR, Dapia S, Calvo E, Largo R, Herrero-Beaumont G: Subchondral bone microstructural damage by increased remodelling aggravates experimental osteoarthritis preceded by osteoporosis. Arthritis Res Ther 2010, 12:R152.

2. Radin EL, Rose RM: Role of subchondral bone in the initiation and progression of cartilage damage. Clin Orthop Relat Res 1986, 213:34-40.

3. Goldring MB, Goldring SR: Articular cartilage and subchondral bone in the pathogenesis of osteoarthritis. Ann N Y Acad Sci 2010, 1192:230-237.

4. Goldring SR: Role of bone in osteoarthritis pathogenesis. Med Clin North Am 2009, 93:25-35, XV.

5. Burr DB: Anatomy and physiology of the mineralized tissues: role in the pathogenesis of osteoarthrosis. Osteoarthritis Cartilage 2004, 12(Suppl A): S20-S30.

6. Karvonen RL, Miller PR, Nelson DA, Granda JL, Fernandez-Madrid F: Periarticular osteoporosis in osteoarthritis of the knee. J Rheumatol 1998, 25:2187-2194.

7. Buckland-Wright C: Subchondral bone changes in hand and knee osteoarthritis detected by radiography. Osteoarthritis Cartilage 2004, 12(Suppl A):S10-S19.

8. Day JS, Ding M, van der Linden JC, Hvid I, Sumner DR, Weinans H: A decreased subchondral trabecular bone tissue elastic modulus is associated with pre-arthritic cartilage damage. J Orthop Res 2001, 19:914-918.

9. Sniekers $Y H$, Intema F, Lafeber FP, van Osch GJ, van Leeuwen JP, Weinans $H$, Mastbergen SC: A role for subchondral bone changes in the process of osteoarthritis; a micro-CT study of two canine models. BMC Musculoskelet Disord 2008, 9:20.

10. Ham KD, Carlson CS: Effects of estrogen replacement therapy on bone turnover in subchondral bone and epiphyseal metaphyseal cancellous bone of ovariectomized cynomolgus monkeys. J Bone Miner Res 2004, 19:823-829.

doi:10.1186/ar3135

Cite this article as: Goldring SR, Goldring MB: Bone and cartilage in osteoarthritis: is what's best for one good or bad for the other? Arthritis Research \& Therapy 2010, 12:143. 